\title{
Testing additive integrality gaps
}

\author{
Friedrich Eisenbrand* \\ Nicolai Hähnle ${ }^{\dagger}$ \\ Dömötör Pálvölgyi \\ Gennady Shmonin ${ }^{\S}$
}

\begin{abstract}
We consider the problem of testing whether the maximum additive integrality gap of a family of integer programs in standard form is bounded by a given constant. This can be viewed as a generalization of the integer rounding property, which can be tested in polynomial time if the number of constraints is fixed. It turns out that this generalization is NP-hard even if the number of constraints is fixed. However, if, in addition, the objective is the all-one vector, then one can test in polynomial time whether the additive gap is bounded by a constant.
\end{abstract}

\section{Introduction}

Linear programming is a very successful tool for solving combinatorial optimization problems that can be modeled as an integer linear program

$$
\min \left\{c^{\mathrm{T}} x: A x=b, x \geqslant 0 \text { integral }\right\},
$$

where $A=\left(a_{i j}\right)$ is a $d \times n$ integral matrix of full rowrank, $b$ is a $d$-element and $c$ is an $n$-element integral vector, respectively. Common to most techniques is solving the linear programming relaxation

$$
\min \left\{c^{\mathrm{T}} x: A x=b, x \geqslant 0\right\} .
$$

and constructing a feasible integer solution from this fractional solution.

Some combinatorial optimization problems like bipartite matching or minimum cost network flows have the property that the matrix $A$ in the integer programming model (1.1) is unimodular, which means that each $d \times d$ submatrix of $A$ has determinant $0, \pm 1$. In this case, the integer program (1.1) can be solved exactly with linear programming since an optimum extreme point of the linear programming problem is integral. This is a property of the matrix $A$. More precisely all extreme points of the polyhedron $\left\{x \in \mathbb{R}^{n}: A x=b, x \geqslant 0\right\}$ are

\footnotetext{
*Ecole Polytechnique Fédérale de Lausanne, Switzerland. Email: friedrich.eisenbrand@epfl.ch

${ }^{\dagger}$ Ecole Polytechnique Fédérale de Lausanne, Switzerland. Email: nicolai.haehnle@epfl.ch

${ }^{\ddagger}$ Ecole Polytechnique Fédérale de Lausanne, Switzerland. Email: doemoetoer.palvoelgyi@epfl.ch

$\S$ Ecole Polytechnique Fédérale de Lausanne, Switzerland. Email: gennady.shmonin@epfl.ch
}

integral for each integral $b$ if and only if the matrix $A$ is unimodular [20]. This is also related to the study of totally unimodular matrices and the Hoffman-Kruskal theorem [8], see e.g. [16]. Seymour [17] showed that totally unimodular matrices, and consequently, also unimodular matrices can be recognized in polynomial time. Thus this convenient property of combinatorial optimization problems having integral linear programming relaxations can be efficiently recognized.

If the linear programming relaxation does not immediately yield an integral optimum solution, one rounds this fractional solution to a feasible integral solution. This paradigm has been very successfully applied in the area of approximation algorithms, see e.g. [19]. As the solution which is thereby found is compared to the solution of the linear programming relaxation, the approximation guarantee of the algorithm depends crucially on the integrality gap of the linear programming relaxation. In this paper we consider additive integrality gaps, i.e., the difference between the optimum solution value of the combinatorial optimization problem and its linear relaxation.

The integer program (1.1) is defined by three parameters: the matrix $A$, the objective vector $c$, and the right-hand side $b$. If the optimum value of the relaxation (1.2) is strictly smaller than the optimum value of the integer program, then this difference can be made arbitrarily large by scaling the objective function vector $c$ with a constant. This shows that, while unimodularity is a sufficient condition on the matrix $A$ yielding integer polyhedra regardless of the objective function vector and the right-hand side, one needs to take the objective function vector into account if one wants to have a similar notion for additive integrality gaps.

The tuple $(A, c)$ has the the integer rounding property [1] if

$$
\begin{aligned}
\min \left\{c^{\mathrm{T}} x: A x=b, x \geqslant\right. & 0 \text { integral }\}= \\
& \left\lceil\min \left\{c^{\mathrm{T}} x: A x=b, x \geqslant 0\right\}\right\rceil
\end{aligned}
$$

for each $b$ such that the linear programming relaxation is feasible and bounded. We shall always assume that the system $y^{\mathrm{T}} A \leqslant c^{\mathrm{T}}$ has a solution; then both the integer program (1.1) and the associated linear programming relaxation (1.2) are not unbounded for all $b$. 
There are many examples of tuples $(A, c)$ stemming from combinatorial optimization problems that have the integer rounding property $[1,5,18,21]$. In this case the integral problem can also be solved exactly by linear programming techniques, see e.g. [15, Theorem 22.15] due to the relation of the integer rounding property with the notions of a Hilbert basis and total dual integrality, which we briefly describe.

The integral vectors $a_{1}, a_{2}, \ldots, a_{n}$ in $\mathbb{R}^{d}$ form a Hilbert basis if every integral vector in the cone generated by $a_{1}, a_{2}, \ldots, a_{n}$ can be expressed as an integral non-negative linear combination of $a_{1}, a_{2}, \ldots, a_{n}$. Giles and Orlin [6] showed that the tuple $(A, c)$ has the integer rounding property if and only if the columns of the matrix

$$
\left(\begin{array}{ll}
1 & c^{\mathrm{T}} \\
0 & A
\end{array}\right)
$$

form a Hilbert basis. The question now arises, whether this property can also be tested in polynomial time, like it is the case for total unimodularity. Pap [13] recently proved that the recognition of Hilbert bases is coNPcomplete, see also [3]. Via the result of Giles and Orlin this also means that testing whether a tuple $(A, c)$ has the integer rounding property is co-NP complete.

However, if $d$ (the number of rows of $A$ ) is fixed, then one can test in polynomial time whether a given family of integer programs has the integer rounding property, by using the algorithm of Cook et al. [2] for recognizing Hilbert bases in fixed dimension.

Contribution of this paper In this paper we consider a generalization of the integer rounding property. We say that $(A, c)$ has the additive integrality gap of at most $\gamma$ if

$$
\begin{aligned}
\min \left\{c^{\mathrm{T}} x\right. & : A x=b, x \geqslant 0 \text { integral }\} \\
& \leqslant\left\lceil\min \left\{c^{\mathrm{T}} x: A x=b, x \geqslant 0\right\}\right\rceil+\gamma,
\end{aligned}
$$

for each $b$ for which the linear programming relaxation (1.2) is feasible. If there is a $b$ for which (1.2) is feasible but $(1.1)$ is infeasible, then $(A, c)$ has infinite integrality gap.

For $\gamma=0$ this is exactly the integer rounding property. Our main results are as follows.

(a) It is NP-hard to test whether $(A, c)$ has additive gap of at most $\gamma$ even if $d$ is fixed and $\gamma=1$. This is in contrast to the integer rounding property $(\gamma=0)$, which can be tested in polynomial time if $d$ is fixed.

(b) For fixed $d$ and $\gamma$, there exists a polynomial algorithm which tests whether $(A, \mathbf{1})$ has additive gap of at most $\gamma$. We use $\mathbf{1}$ to denote the all-one vector.
Many combinatorial optimization problems of setpacking or set-partitioning nature have objective function $\mathbf{1}^{\mathrm{T}} x$ which makes the result (b) a relevant extension of the test for the integer rounding property of combinatorial optimization problems. While the result (a) seems surprising at first, we describe our algorithm which solves (b) such that it runs in polynomial time under a more general assumption than all-one objective function. The case $\gamma=0$ also falls under this restriction and thus our algorithm can be interpreted as a generalization of the Hilbert basis test in fixed dimension of Cook et al. [2].

Here is a brief outline on how these results are proved. First we extend the notion of a Hilbert basis to a so-called $\gamma$-relaxed Hilbert basis. We prove that the tuple $(A, c)$ has additive integrality gap at most $\gamma$ if and only if the columns of the matrix (1.3) form a $\gamma$ relaxed Hilbert basis. Then we consider the recognition problem for $\gamma$-relaxed Hilbert bases in fixed dimension. Via a reduction from the Frobenius problem we show that it is NP-hard to decide whether $n$ positive integers form a 1-relaxed Hilbert basis. This is then extended to the result (a).

The positive result (b) is obtained via the construction of a hyperplane arrangement and the use of an algorithm for integer programming in fixed dimension to identify those right-hand sides, for which the gap is larger than $\gamma$.

Related work Hoşten and Sturmfels [7] considered a similar recognition problem, where the right-hand side $b$ ranges over all integral vectors, for which the integer program (1.1) is feasible (in contrast, we consider integral vectors $b$, for which the associated linear program is feasible; thus the additive integrality gap may happen to be infinite). They designed an algorithm that tests if the additive integrality gap is bounded by $\gamma$ in polynomial time if both the number of constraints $d$ and the number of variables $n$ are fixed. Eisenbrand and Shmonin [4] generalized this result, presenting an algorithm that tests the additive integrality gap for families of integer programs of the form

$$
\max \left\{c^{\mathrm{T}} x: A x \leqslant b, x \text { integral }\right\},
$$

where $b$ ranges over all vectors, for which the integer program is feasible. This algorithm runs in polynomial time assuming only that the number of variables $n$ is fixed. These algorithms are based on an algorithm of Kannan $[9,10]$ to decide general $\forall \exists$-statements.

\section{Some basic definitions and notation Let $a_{1}, a_{2}, \ldots, a_{n}$ be vectors in the Euclidean space}


$\mathbb{R}^{d}$. The cone generated by these vectors is the set

$$
\text { cone }\left(a_{1}, a_{2}, \ldots, a_{n}\right):=\left\{\sum_{i=1}^{n} \lambda_{i} a_{i}: \lambda_{i} \geqslant 0 \text { for all } i\right\} \text {. }
$$

If $b \in \operatorname{cone}\left(a_{1}, a_{2}, \ldots, a_{n}\right)$, then we say that $b$ can be expressed as a non-negative combination of vectors $a_{1}, a_{2}, \ldots, a_{n}$. The integer cone generated by $a_{1}, a_{2}, \ldots, a_{n}$ is the semigroup

$$
\begin{aligned}
& \text { int.cone }\left(a_{1}, a_{2}, \ldots, a_{n}\right):= \\
& \qquad\left\{\sum_{i=1}^{n} \lambda_{i} a_{i}: \lambda_{i} \geqslant 0 \text { integer for all } i\right\} .
\end{aligned}
$$

We say that $b \in$ int.cone $\left(a_{1}, a_{2}, \ldots, a_{n}\right)$ can be expressed as an integral non-negative combination of vectors $a_{1}, a_{2}, \ldots, a_{n}$. The integral vectors $a_{1}, a_{2}, \ldots, a_{n}$ in $\mathbb{R}^{d}$ form a Hilbert basis if

$$
\operatorname{cone}\left(a_{1}, a_{2}, \ldots, a_{n}\right) \cap \mathbb{Z}^{d}=\operatorname{int} . \operatorname{cone}\left(a_{1}, a_{2}, \ldots, a_{n}\right) \text {. }
$$

A cone is pointed if it has an extreme point, or equivalently, there is a vector $d$ such that $d^{\mathrm{T}} x>0$ for all non-zero vectors $x$ from the cone. It is known that every pointed finitely generated rational cone has a unique minimal Hilbert basis; see e.g. [15].

\section{Relaxed Hilbert bases}

In this section we provide an analogous result to the one of Giles and Orlin [6] for bounded integrality gap. For this, we need to generalize the notion of a Hilbert basis.

Let $a_{0}, a_{1}, \ldots, a_{n}$ be integral vectors in $\mathbb{R}^{d}$ and let $\gamma$ be a non-negative integer. We say that the vectors $a_{0}, a_{1}, \ldots, a_{n}$ form a $\gamma$-relaxed Hilbert basis with respect to $a_{0}$ if every integral vector $b$ in $\operatorname{cone}\left(a_{0}, a_{1}, \ldots, a_{n}\right)$ can be written as an integral combination

$b=\sum_{i=0}^{n} \lambda_{i} a_{i}, \lambda_{0}+\gamma \geqslant 0$ and $\lambda_{i} \geqslant 0$ for $i=1,2, \ldots, n$.

In other words, we require that at least one of the vectors

$$
b, b+a_{0}, b+2 a_{0}, \ldots, b+\gamma a_{0}
$$

belongs to int.cone $\left(a_{0}, a_{1}, \ldots, a_{n}\right)$. Moreover, if $b+i a_{0}$ belongs to int.cone $\left(a_{0}, a_{1}, \ldots, a_{n}\right)$ for some integer $i \leqslant$ $\gamma$, then $b+\gamma a_{0}$ also belongs to int.cone $\left(a_{0}, a_{1}, \ldots, a_{n}\right)$. Thus, vectors $a_{0}, a_{1}, \ldots, a_{n}$ form a $\gamma$-relaxed Hilbert basis if and only if

$$
b+\gamma a_{0} \in \text { int.cone }\left(a_{0}, a_{1}, \ldots, a_{n}\right)
$$

whenever $b \in \operatorname{cone}\left(a_{0}, a_{1}, \ldots, a_{n}\right) \cap \mathbb{Z}^{d}$.
THEOREM 2.1. Let $A$ be an integral matrix and let $c$ be an integral vector. The family of integer programs

$$
\min \left\{c^{\mathrm{T}} x: A x=b, x \geqslant 0 \text { integral }\right\}
$$

has the additive integrality gap of at most $\gamma$ if and only if the columns of the matrix

$$
\left(\begin{array}{ll}
1 & c^{\mathrm{T}} \\
0 & A
\end{array}\right)
$$

form a $\gamma$-relaxed Hilbert basis with respect to the first column.

Proof. Suppose that the additive integrality gap of (2.5) is at most $\gamma$. Let $\left(\begin{array}{l}\alpha \\ b\end{array}\right)$ be an arbitrary integral vector from the cone generated by the columns of the matrix (2.6). We have to show that $\left(\begin{array}{c}\alpha+\gamma \\ b\end{array}\right)$ is in the integer cone generated by the columns of the matrix (2.6).

One has $\alpha=\hat{\xi}+c^{\mathrm{T}} \hat{x}$ and $b=A \hat{x}$ for some number $\hat{\xi} \geqslant 0$ and some vector $\hat{x} \geqslant 0$. It follows that

$$
\min \left\{c^{\mathrm{T}} x: A x=b, x \geqslant 0\right\} \leqslant c^{\mathrm{T}} \hat{x} \leqslant \alpha .
$$

Since the additive integrality gap of the integer program $(2.5)$ is at most $\gamma$, the optimum value $\beta$ of $(2.5)$ is at most $\alpha+\gamma$. Thus the vector $\left(\begin{array}{l}\beta \\ b\end{array}\right)$ belongs to the integer cone generated by the columns of matrix (2.6). Since the vector $\left(\begin{array}{l}1 \\ 0\end{array}\right)$ belongs to $(2.6)$, so does $\left(\begin{array}{c}\alpha+\gamma \\ b\end{array}\right)$.

Conversely, suppose that the columns of the matrix (2.6) form a $\gamma$-relaxed Hilbert basis and let $b$ be an integral vector such that the system $A x=b$ has a non-negative solution. Let $\alpha$ denote the optimum value of the linear program

$$
\min \left\{c^{\mathrm{T}} x: A x=b, x \geqslant 0\right\} .
$$

Then $\alpha=c^{\mathrm{T}} \hat{x}$ and $b=A \hat{x}$ for some vector $\hat{x} \geqslant$ 0 . Consequently, the integral vector $\left(\begin{array}{c}\lceil\alpha\rceil \\ b\end{array}\right)$ belongs to the cone generated by the columns of matrix (2.6). Therefore, there is a vector $\left(\begin{array}{c}\beta \\ b\end{array}\right)$, with $\beta \leqslant\lceil\alpha\rceil+\gamma$, that belongs to the integer cone generated by the columns of matrix (2.6). But the latter implies that the optimum value of the integer program $(2.5)$ is at most $\beta \leqslant\lceil\alpha\rceil+\gamma$.

It is easy to see that the cone generated by the columns of the matrix (2.6) is pointed if and only if the system $y^{\mathrm{T}} A<c$ has a solution. We shall need the following two lemmas in our recognition algorithm in the last section of this paper. The first lemma is a generalization of a statement about classical Hilbert bases $(\gamma=0)$ by Cook et al. [2]. It is proved in a similar way.

LEMMA 2.1. Let $a_{0}, a_{1}, \ldots, a_{n}$ be integral vectors in $\mathbb{R}^{d}$ such that the cone cone $\left(a_{0}, a_{1}, \ldots, a_{n}\right)$ is pointed. Then $a_{0}, a_{1}, \ldots, a_{n}$ do not form a $\gamma$-relaxed Hilbert basis with 
respect to $a_{0}$ if and only if there is an integral vector $b \in \operatorname{cone}\left(a_{0}, a_{1}, \ldots, a_{n}\right)$ such that

$b-a_{j} \notin$ cone $\left(a_{0}, a_{1}, \ldots, a_{n}\right) \quad$ for each $j=0,1, \ldots, n$,

and

$$
b+\gamma a_{0} \notin \text { int.cone }\left(a_{0}, a_{1}, \ldots, a_{n}\right) .
$$

Proof. Sufficiency is clear. Suppose that $a_{0}, a_{1}, \ldots, a_{n}$ do not form a $\gamma$-relaxed Hilbert basis. Since cone $\left(a_{0}, a_{1}, \ldots, a_{n}\right)$ is pointed, there is a vector $d$ with $d^{\mathrm{T}} a_{j}>0$ for $j=0,1, \ldots, n$. Let $b$ be an integral vector in cone $\left(a_{0}, a_{1}, \ldots, a_{n}\right)$ such that $b+$ $\gamma a_{0} \notin$ int.cone $\left(a_{0}, a_{1}, \ldots, a_{n}\right)$. Among all possible $b$ 's we choose one that minimizes $d^{\mathrm{T}} b$. Since $d^{\mathrm{T}}(b-$ $\left.a_{j}\right)<d^{\mathrm{T}} b$ one has, by the minimality of $b, b-a_{j} \notin$ cone $\left(a_{0}, a_{1}, \ldots, a_{n}\right)$ for each $j=0,1, \ldots, n$.

We obtain the following corollary which helps us to restrict the search for vectors $b$ which prove that $a_{0}, a_{1}, \ldots, a_{n}$ is a $\gamma$-relaxed Hilbert bases.

Corollary 2.1. Let $a_{0}, a_{1}, \ldots, a_{n}$ be integral vectors in $\mathbb{R}^{d}$ such that cone $\left(a_{0}, a_{1}, \ldots, a_{n}\right)$ is a pointed cone. Then $a_{0}, a_{1}, \ldots, a_{n}$ do not form a $\gamma$-relaxed Hilbert basis with respect to $a_{0}$ if and only if there is an integral vector $b$ in cone $\left(a_{0}, a_{1}, \ldots, a_{n}\right)$ such that

$$
b+\gamma a_{0} \notin \operatorname{int} . \operatorname{cone}\left(a_{0}, a_{1}, \ldots, a_{n}\right) .
$$

and

$$
\max \left\{\mathbf{1}^{\mathrm{T}} x: A x=b, x \geqslant 0\right\}<d,
$$

where $A=\left(\begin{array}{llll}a_{0} & a_{1} & \ldots & a_{n}\end{array}\right)$ is the matrix composed of vectors $a_{0}, a_{1}, \ldots, a_{n}$ as columns.

Proof. Sufficiency is again clear. Let $b$ be a vector satisfying the conditions of Lemma 2.1. Consider the linear program

$$
\max \left\{\mathbf{1}^{\mathrm{T}} x: A x=b, x \geqslant 0\right\} .
$$

From the theory of linear programming, we know that there is a basic optimum solution of the above linear program having at most $d$ non-zero components, i.e.,

$$
\sum_{j=1}^{d} x_{i_{j}} a_{i_{j}}=b, \quad x_{i_{j}} \geqslant 0
$$

for some indices $i_{1}, i_{2}, \ldots, i_{d}$. Then $x_{i_{j}}<1$ for all $j=1,2, \ldots, d$, as otherwise $b-a_{i_{j}}$ would belong to cone $\left(a_{0}, a_{1}, \ldots, a_{n}\right)$, which contradicts the choice of $b$. But then

$$
\mathbf{1}^{\mathrm{T}} x=\sum_{j=1}^{d} x_{i_{j}}<d .
$$

\section{$3 \quad$ Hardness of gap testing}

In this section we show that it is NP-hard to test whether $(A, c)$ has integrality gap at most 1 , even if $A$ has only one row. Since this is equivalent to deciding if the columns of the matrix (2.6) form a 1-relaxed Hilbert basis with respect to the first column we first consider a general problem of recognizing 1-relaxed Hilbert bases.

We proceed by a reduction from the Frobenius problem (also known as the coin problem). Let $\alpha_{1}, \alpha_{2}, \ldots, \alpha_{n}$ be positive integers such that their greatest common divisor is 1 . The smallest integer $t$, such that every integer $z>t$ is an element of int.cone $\left(\alpha_{1}, \alpha_{2}, \ldots, \alpha_{n}\right)$, is called the Frobenius number and is denoted by $F\left(\alpha_{1}, \alpha_{2}, \ldots, \alpha_{n}\right)$. The condition $\operatorname{gcd}\left(\alpha_{1}, \alpha_{2}, \ldots, \alpha_{n}\right)=1$ implies that the Frobenius number is finite. The Frobenius problem is to decide whether

$$
F\left(\alpha_{1}, \alpha_{2}, \ldots, \alpha_{n}\right)<t,
$$

for given positive integers $\alpha_{1}, \alpha_{2}, \ldots, \alpha_{n}$ and $t$. Kannan [10] showed that the Frobenius problem can be solved in polynomial time, if $n$ is fixed. For general $n$ it is known to be NP-hard under Turing reductions [14].

THEOREM 3.1. Testing whether positive integers $a_{0}, a_{1}, \ldots, a_{n}$ form a 1-relaxed Hilbert basis with respect to $a_{0}$ is NP-hard.

Proof. We demonstrate a Karp-reduction from the Frobenius problem. Let $\alpha_{1}, \alpha_{2}, \ldots, \alpha_{n}$ and $t$ be positive integers defining an instance of the Frobenius problem and consider the integers

$$
a_{0}=4 t, a_{1}=2 \alpha_{1}, \ldots, a_{n}=2 \alpha_{n}, a_{n+1}=2 t+1
$$

We claim that the integers (3.7) form a 1-relaxed Hilbert basis with respect to $a_{0}=4 t$ if and only if $F\left(\alpha_{1}, \alpha_{2}, \ldots, \alpha_{n}\right)<t$. Observe that the cone generated by (3.7) is exactly the set of all non-negative numbers.

Suppose that $F\left(\alpha_{1}, \alpha_{2}, \ldots, \alpha_{n}\right)<t$. Then every even integer $z \geqslant 2 t$ can be expressed as an integral nonnegative combination of $2 \alpha_{1}, 2 \alpha_{2}, \ldots, 2 \alpha_{n}$,

$$
\begin{aligned}
& z \in \text { int.cone }\left(2 \alpha_{1}, 2 \alpha_{2}, \ldots, 2 \alpha_{n}\right) \\
& \text { for all } z \geqslant 2 t \text { even. }
\end{aligned}
$$

If $0 \leqslant z<2 t$ is an even integer, then $z+4 t>2 t$ is also even, and (3.8) implies

$$
z+4 t \in \text { int.cone }\left(2 \alpha_{1}, 2 \alpha_{2}, \ldots, 2 \alpha_{n}\right)
$$

for all $0 \leqslant z<2 t$ even.

If $z \geqslant 0$ is odd, then $z+4 t-(2 t+1) \geqslant 2 t$ is even and thus an element of int.cone $\left(2 \alpha_{1}, 2 \alpha_{2}, \ldots, 2 \alpha_{n}\right)$. 
Thus for each odd $z \geqslant 0$ one has $z+4 t \in$ int.cone $\left(2 \alpha_{1}, 2 \alpha_{2}, \ldots, 2 \alpha_{n}, 2 t+1\right)$. This shows that (3.7) is a 1-relaxed Hilbert basis.

For the converse we need to show that each $z \geqslant t$ is an element of int.cone $\left(\alpha_{1}, \alpha_{2}, \ldots, \alpha_{n}\right)$. It is enough to show this for each $z$ with $t \leqslant z<2 t$ since each integer $x \geqslant 2 t$ can be written as $x=q t+\ell$ with $q \geqslant 0$ and $t \leqslant \ell<2 t$.

We can write $z$ as $z=t+y$ with $0 \leqslant y \leqslant t-1$. Since $2 y+1$ is odd, one has

$$
2 y+1 \notin \text { int.cone }\left(4 t, 2 \alpha_{1}, 2 \alpha_{2} \ldots, 2 \alpha_{n}\right) .
$$

However, we have

$$
(2 y+1)+4 t \in \text { int.cone }\left(4 t, 2 \alpha_{1}, 2 \alpha_{2} \ldots, 2 \alpha_{n}, 2 t+1\right)
$$

since $4 t, 2 \alpha_{1}, 2 \alpha_{2}, \ldots, 2 \alpha_{n}, 2 t+1$ is a 1 -relaxed Hilbert basis. But $4 t$ cannot have a positive coefficient in a non-negative integral combination of $4 t, 2 \alpha_{1}, 2 \alpha_{2}$, $\ldots, 2 \alpha_{n}, 2 t+1$ yielding $(2 y+1)+4 t$. This implies $(2 y+1)+4 t \in$ int.cone $\left(2 \alpha_{1}, 2 \alpha_{2}, \ldots, 2 \alpha_{n}, 2 t+1\right)$. The coefficient of $2 t+1$ must be one. It cannot be two, since the result would be an even number and it cannot be larger than two since the outcome would be more than $6 t$. Thus $(2 y+1)+4 t-(2 t+1) \in$ int.cone $\left(2 \alpha_{1}, 2 \alpha_{2}, \ldots, 2 \alpha_{n}\right)$. But $2 y+2 t=z$ which shows that $z \in$ int.cone $\left(\alpha_{1}, \alpha_{2}, \ldots, \alpha_{n}\right)$.

The result of Theorem 3.1 is not directly applicable to the problem of recognizing additive integrality gaps. Now, we modify the reduction to settle the complexity of recognizing relaxed Hilbert bases, provided that the input vectors are of the form (2.6).

THEOREM 3.2. Testing whether a family of integer programs (2.5) has the additive integrality gap of at most 1 is NP-hard, even if there is only one non-trivial constraint.

Proof. Due to Theorem 2.1, we need to show that it remains NP-hard to test whether a set of vectors is a 1-relaxed Hilbert basis, where $a_{0}$ is a unit vector and the cone that the set of vectors generate is pointed.

We observe that for any unimodular matrix $U$, the vectors $a_{0}, a_{1}, \ldots, a_{n}$ form a $\gamma$-relaxed Hilbert basis if and only if $U a_{0}, U a_{1}, \ldots, U a_{n}$ do. Consequently, it suffices to show hardness when $a_{0}$ and $a_{1}$ are required to form a unimodular matrix $\left(\begin{array}{ll}a_{0} & a_{1}\end{array}\right)$. By applying an appropriate unimodular transformation, we can then transform $a_{0}$ and $a_{1}$ into the unit vectors $e_{1}=U a_{0}$ and $e_{2}=U a_{1}$.

The reduction is again from the Frobenius problem and, in fact, very similar to that in the proof of Theorem 3.1. Given positive integers $\alpha_{1}, \alpha_{2}, \ldots, \alpha_{n}$ and $t$ defining an instance of the Frobenius problem, we construct an instance of the relaxed Hilbert basis recognition problem as follows:

$$
\begin{aligned}
& a_{0}=\left(\begin{array}{c}
1 \\
4 t
\end{array}\right), a_{1}=\left(\begin{array}{c}
2 \\
8 t-1
\end{array}\right),\left(\begin{array}{l}
1 \\
0
\end{array}\right) \\
& \left(\begin{array}{c}
0 \\
2 \alpha_{1}
\end{array}\right),\left(\begin{array}{c}
0 \\
2 \alpha_{2}
\end{array}\right), \ldots,\left(\begin{array}{c}
0 \\
2 \alpha_{n}
\end{array}\right),\left(\begin{array}{c}
0 \\
2 t+1
\end{array}\right) .
\end{aligned}
$$

We claim that $F\left(\alpha_{1}, \alpha_{2}, \ldots, \alpha_{n}\right)<t$ if and only if the vectors (3.9) form a 1-relaxed Hilbert basis with respect to $a_{0}$. It is easy to see that the cone generated by (3.9) is exactly the set of all non-negative vectors.

Suppose that $F\left(\alpha_{1}, \alpha_{2}, \ldots, \alpha_{n}\right)<t$. Similar to the proof of Theorem 3.1, we can show that for any integral vector $v=\left(\begin{array}{l}0 \\ z\end{array}\right)$, at least one of the following holds:

$v=\left(\begin{array}{l}0 \\ z\end{array}\right) \in$ int.cone $\left(\left(\begin{array}{c}0 \\ 2 \alpha_{1}\end{array}\right), \ldots,\left(\begin{array}{c}0 \\ 2 \alpha_{n}\end{array}\right),\left(\begin{array}{c}0 \\ 2 t+1\end{array}\right)\right)$

or

$$
\begin{aligned}
& v+a_{0}=\left(\begin{array}{c}
1 \\
z+4 t
\end{array}\right) \in \\
& \text { int.cone }\left(\left(\begin{array}{l}
1 \\
0
\end{array}\right),\left(\begin{array}{c}
0 \\
2 \alpha_{1}
\end{array}\right), \ldots,\left(\begin{array}{c}
0 \\
2 \alpha_{n}
\end{array}\right),\left(\begin{array}{c}
0 \\
2 t+1
\end{array}\right)\right) .
\end{aligned}
$$

Since the unit vector $\left(\begin{array}{l}1 \\ 0\end{array}\right)$ belongs to the set $(3.9)$, this implies the claim for all integral non-negative vectors.

Suppose that the vectors (3.9) form a 1-relaxed Hilbert basis with respect to $a_{0}$. We need to show that every integer $z \geqslant t$ belongs to int.cone $\left(\alpha_{1}, \alpha_{2}, \ldots, \alpha_{n}\right)$. Again, we may restrict our attention to integers $t \leqslant z \leqslant$ $2 t-1$ only. Set $z=t+y$, then $0 \leqslant y \leqslant t-1$. The vector $v=\left(\begin{array}{c}0 \\ 2 y+1\end{array}\right)$ does not belong to the integer cone generated by $(3.9)$, since $2 y+1$ is odd and $2 y+1<2 t+1$. We conclude that $v+a_{0}=\left(\begin{array}{c}2 y+1+4 t \\ 2\end{array}\right)$ is in the integer cone of (3.9), i.e.,

$$
\begin{gathered}
v+a_{0}=\left(\begin{array}{c}
1 \\
2 y+1+4 t
\end{array}\right)= \\
\lambda_{0}\left(\begin{array}{c}
1 \\
4 t
\end{array}\right)+\sum_{j=1}^{n} \lambda_{j}\left(\begin{array}{c}
0 \\
2 \alpha_{j}
\end{array}\right)+ \\
\xi\left(\begin{array}{c}
2 \\
8 t-1
\end{array}\right)+\eta\left(\begin{array}{l}
1 \\
0
\end{array}\right)+\mu\left(\begin{array}{c}
0 \\
2 t+1
\end{array}\right)
\end{gathered}
$$

for some non-negative integers $\lambda_{0}, \lambda_{1}, \ldots, \lambda_{n}, \xi, \eta$ and $\mu$. Clearly, $\xi=0$. Moreover, $\lambda_{0}=0$, since $v$ itself does not belong to the integer cone of (3.9). Consequently, $\eta=1$, as $\left(\begin{array}{l}1 \\ 0\end{array}\right)$ is the only remaining vector with nonzero in the first component. Thus, we are left with the second component of $v+a_{0}$ only:

$$
2 y+1+4 t=\sum_{j=1}^{n} \lambda_{j}\left(2 \alpha_{j}\right)+\mu(2 t+1) .
$$


As in the proof of Theorem 3.1 we condlude that $\mu=1$, which implies

$$
2 z=2 t+2 y \in \text { int.cone }\left(2 \alpha_{1}, 2 \alpha_{2}, \ldots, 2 \alpha_{n}\right) .
$$

Finally, we observe that the vectors $a_{0}$ and $a_{1}$ in (3.9) form a unimodular matrix $\left(\begin{array}{ll}a_{0} & a_{1}\end{array}\right)$, as required.

\section{The case of all-one objectives}

In this section we provide a proof of the following theorem.

Theorem 4.1. For fixed $\gamma$ and $d$, there is a polynomial algorithm which tests whether $(A, \mathbf{1})$ has integrality gap at most $\gamma$.

In fact, we provide an algorithm that recognizes whether a given set of vectors $a_{0}, a_{1}, \ldots, a_{n} \in \mathbb{Z}^{d}$, generating a pointed cone, is a $\gamma$-relaxed Hilbert basis in polynomial time, if the matrix $A=\left(a_{0}, a_{1}, \ldots, a_{n}\right)$ satisfies the following condition.

Condition 4.1. There is a constant $f(\gamma, d)$ depending only on $\gamma$ and $d$ such that

$$
\max \left\{\mathbf{1}^{\mathrm{T}} x: A x=b+\gamma a_{0}, x \geqslant 0\right\} \leqslant d+f(\gamma, d)
$$

whenever

$$
\begin{aligned}
& b \in \operatorname{cone}\left(a_{0}, a_{1}, \ldots, a_{n}\right) \text { and } \\
& \qquad \max \left\{\mathbf{1}^{\mathrm{T}} x: A x=b, x \geqslant 0\right\} \leqslant d .
\end{aligned}
$$

Notice that since the cone is assumed to be pointed, both maxima in Condition 4.1 exist whenever the system $A x=b, x \geqslant 0$ has a solution. The condition holds, in particular, for the columns of the matrix (2.6) when $c$ is the all-one vector 1 . In this case the first row of the matrix $A$ in (4.10) is also the all-one vector and $a_{0}$ is the first unit vector. Therefore, the cone generated by the columns of $\mathrm{A}$ is pointed and (4.10) implies

$$
\max \left\{\mathbf{1}^{\mathrm{T}} x: A x=b+\gamma a_{0}, x \geqslant 0\right\} \leqslant d+\gamma .
$$

Notice that Condition 4.1 trivially holds if $\gamma=0$. So our algorithm generalizes the one of Cook et al. [2] to test classical Hilbert bases.

We now make use of Corollary 2.1: How can we find an integral $b \in \operatorname{cone}\left(a_{0}, a_{1}, \ldots, a_{n}\right)$ such that

$$
\begin{aligned}
& \max \left\{\mathbf{1}^{\mathrm{T}} x: A x=b, x \geqslant 0\right\}<d \text { and } \\
& b+\gamma a_{0} \notin \text { int.cone }\left(a_{0}, a_{1}, \ldots, a_{n}\right) ?
\end{aligned}
$$

Let $Q \subseteq \mathbb{R}^{d}$ denote the set of all vectors $b$ that satisfy (4.10). This set $Q$ can be efficiently stored and computed, since $d$ is fixed. This is shown in the next

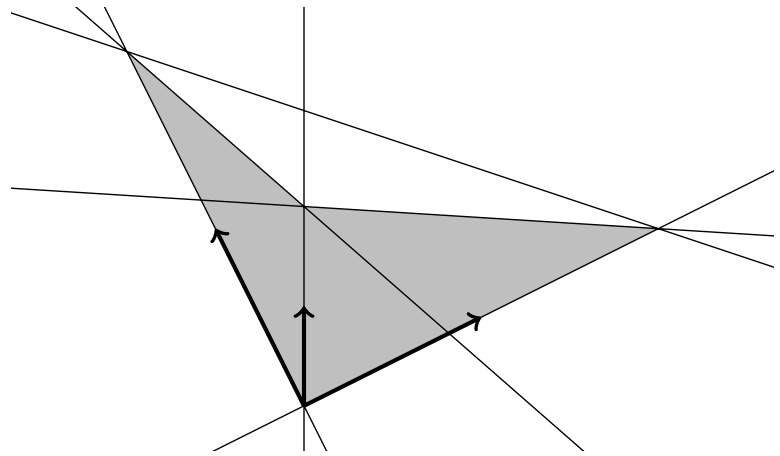

Figure 1: The hyperplane arrangement of Lemma 4.1 for a cone generated by three vectors. The region $Q$ is shaded gray.

lemma, following mainly from the hyperplane arrangement argument that polynomially many hyperplanes partition the whole space into polynomially many cells; see e.g. [12].

Lemma 4.1. For fixed $d$, there is a polynomial algorithm that computes polyhedra $Q_{1}, Q_{2}, \ldots, Q_{t}$ such that $Q=Q_{1} \cup Q_{2} \cup \ldots \cup Q_{t}$.

Proof. From the theory of linear programming we know that for every vector $b$ for which the linear program

$$
\max \left\{\mathbf{1}^{\mathrm{T}} x: A x=b, x \geqslant 0\right\}
$$

is feasible and bounded, there is a basic optimal solution. Equivalently, there is a $d \times d$-submatrix of $A$, say $A^{\prime}$, such that

$$
\begin{aligned}
\max \left\{\mathbf{1}^{\mathrm{T}} x: A x=b,\right. & \geqslant 0\} \\
& =\max \left\{\mathbf{1}^{\mathrm{T}} x: A^{\prime} x=b, x \geqslant 0\right\} .
\end{aligned}
$$

For fixed $d$, we can enumerate all $d \times d$-submatrices of $A$ in polynomial time, say $A_{1}, A_{2}, \ldots, A_{q}$. For each of these submatrices $A_{i}$, the set of vectors $b$, for which

$$
\max \left\{\mathbf{1}^{\mathrm{T}} x: A_{i} x=b, x \geqslant 0\right\} \leqslant d
$$

is just a simplex $S_{i}$ in $\mathbb{R}^{d}$, and hence can be expressed as an intersection of $d+1$ half-spaces. Furthermore, $d$ of these half-spaces define the (simplicial) cone $C_{i}=$ cone $\left(A_{i}\right)$ of all vectors $b$ for which the above linear program is feasible.

Now, $b \in S_{i}$ belongs to $Q$ if and only if $b \in S_{j}$ for all indices $j$ such that $b \in C_{j}$. If $d$ is fixed, then the arrangement of hyperplanes defining the simplices $S_{i}, \quad i=1 \ldots q$, partition the whole space $\mathbb{R}^{d}$ into polynomially many cells $Q_{1}, Q_{2}, \ldots, Q_{l}$. It is now easy 
to see that if $b \in Q_{i}$ belongs to $Q$, then all $b^{\prime} \in Q_{i}$ belong to $Q$, and vice versa. Thus, a subset of these cells yields a required partition.

We now have to check that for all integral vectors $b$ in $Q$, the vector $b+\gamma a_{0}$ is an integral non-negative combination of $a_{0}, a_{1}, \ldots, a_{n}$. Condition 4.1 implies that $b+\gamma a_{0}$ is a sum of at most $d+f(\gamma, d)$ vectors from $a_{0}, a_{1}, \ldots, a_{n}$ (with repetitions allowed). If $d$ and $\gamma$ are fixed, the number of such sums is bounded from above by $(n+1)^{d+f(\gamma, d)}$ and we can enumerate them all in polynomial time. Thus, we obtain a set, say $V$, of integral vectors that are expressible as the sum of at most $d+f(\gamma, d)$ elements from $a_{0}, a_{1}, \ldots, a_{n}$. Then the set

$$
V^{*}:=\left\{v-\gamma a_{0}: v \in V\right\}
$$

covers all integral vectors in $Q$ if and only if $a_{0}, a_{1}, \ldots, a_{n}$ form a $\gamma$-relaxed Hilbert basis with respect to $a_{0}$. Now, our algorithm searches for an integral vector $b \in Q$ with $b \notin V^{*}$.

LEMMA 4.2. If $d$ and $\gamma$ are fixed, there is a polynomial algorithm that computes open polyhedra $P_{1}, P_{2}, \ldots, P_{k}$ such that for any integral vector $b \in Q, b \notin V^{*}$ if and only if $b \in P_{i}$ for some index $i=1,2, \ldots, k$.

Proof. Since $Q$ is a bounded set, for each $b \in V^{*}$ we can find a hyperplane $H$ such that $b$ is the only integral vector from $Q$ lying on $H_{b}$. Thus, there are polynomially many hyperplanes $H_{1}, H_{2}, \ldots, H_{t}$ such that an integral vector $b \in Q$ belongs to $V^{*}$ if and only if $b \in H_{i}$ for some $i=1,2, \ldots, t$. These hyperplanes partition the whole space $\mathbb{R}^{d}$ into polynomially many cells $P_{1}^{\prime}, P_{2}^{\prime}, \ldots, P_{k}^{\prime}$, computable in polynomial time, where each cell is a polyhedron whose facets lie on some of the hyperplanes $H_{1}, H_{2}, \ldots, H_{t}$. The interiors of these polyhedra yield the desired open polyhedra $P_{1}, P_{2}, \ldots, P_{t}$.

By Lemma 4.2 and 4.1, it is now sufficient to check that the sets $P_{i} \cap Q_{j}$ do not contain integral vectors, for all indices $i$ and $j$. So we get a polynomial number of integer programming problems, each of which has $d$ variables and is therefore solvable in polynomial time if $d$ is fixed [11]. This proves Theorem 4.1.

\section{References}

[1] S. P. Baum and L. E. Trotter, Jr. Integer rounding for polymatroid and branching optimization problems. SIAM Journal on Algebraic and Discrete Methods, 2(4):416-425, 1981.

[2] W. J. Cook, L. Lovász, and A. Schrijver. A polynomialtime test for total dual integrality in fixed dimension. In B. H. Korte and K. Ritter, editors, Mathematical Programming at Oberwolfach II, volume 22 of
Mathematical Programming Study, pages 64-69. NorthHolland, 1984.

[3] G. Ding, L. Feng, and W. Zang. The complexity of recognizing linear systems with certain integrality properties. Mathematical Programming, 114(2):321$334,2008$.

[4] F. Eisenbrand and G. Shmonin. Parametric integer programming in fixed dimension. Mathematics of Operations Research, 33(4):839-850, 2008.

[5] D. Gijswijt. Integer decomposition for polyhedra defined by nearly totally unimodular matrices. SIAM Journal on Discrete Mathematics, 19(3):798-806, 2005.

[6] F. R. Giles and J. B. Orlin. Verifying total dual integrality. Manuscript, 1981.

[7] S. Hoşten and B. Sturmfels. Computing the integer programming gap. Combinatorica, 27(3):367-382, 2007.

[8] A. J. Hoffman and J. B. Kruskal. Integral boundary points of convex polyhedra. In H. W. Kuhn and A. W. Tucker, editors, Linear Inequalities and Related Systems, volume 38 of Annals of Mathematics Studies, pages 223-246. Princeton University Press, 1956.

[9] R. Kannan. Test sets for integer programs, $\forall \exists$ sentences. In W. J. Cook and P. D. Seymour, editors, Polyhedral Combinatorics: Proceedings of a DIMACS Workshop held at the Center for Discrete Mathematics and Theoretical Computer Science, June 12-16, 1989, volume 1 of DIMACS Series in Discrete Mathematics and Theoretical Computer Science, pages 39-47. American Mathematical Society, 1990.

[10] R. Kannan. Lattice translates of a polytope and the Frobenius problem. Combinatorica, 12(2):161-177, 1992.

[11] H. W. Lenstra, Jr. Integer programming with a fixed number of variables. Mathematics of Operations Research, 8(4):538-548, 1983.

[12] J. Matoušek. Lectures on Discrete Geometry, volume 212 of Graduate Texts in Mathematics. SpringerVerlag, 2002.

[13] J. Pap. Recognizing conic TDI systems is hard, 2008. To appear in Mathematical Programming.

[14] J. L. Ramírez Alfonsín. Complexity of the Frobenius problem. Combinatorica, 16(1):143-147, 1996.

[15] A. Schrijver. Theory of Linear and Integer Programming. Wiley-Interscience Series in Discrete Mathematics and Optimization. John Wiley \& Sons, 1986.

[16] A. Schrijver. Combinatorial Optimization: Polyhedra and Efficiency, volume 24 of Algorithms and Combinatorics. Springer-Verlag, 2003.

[17] P. D. Seymour. Decomposition of regular matroids. Journal of Combinatorial Theory, Series B, 28(3):305359, 1980.

[18] S. K. Tipnis and L. E. Trotter, Jr. Node-packing problems with integer rounding properties. SIAM Journal on Discrete Mathematics, 2(3):407-412, 1989.

[19] V. V. Vazirani. Approximation Algorithms. SpringerVerlag, 2001.

[20] A. F. Veinott, Jr. and G. B. Dantzig. Integral extreme 


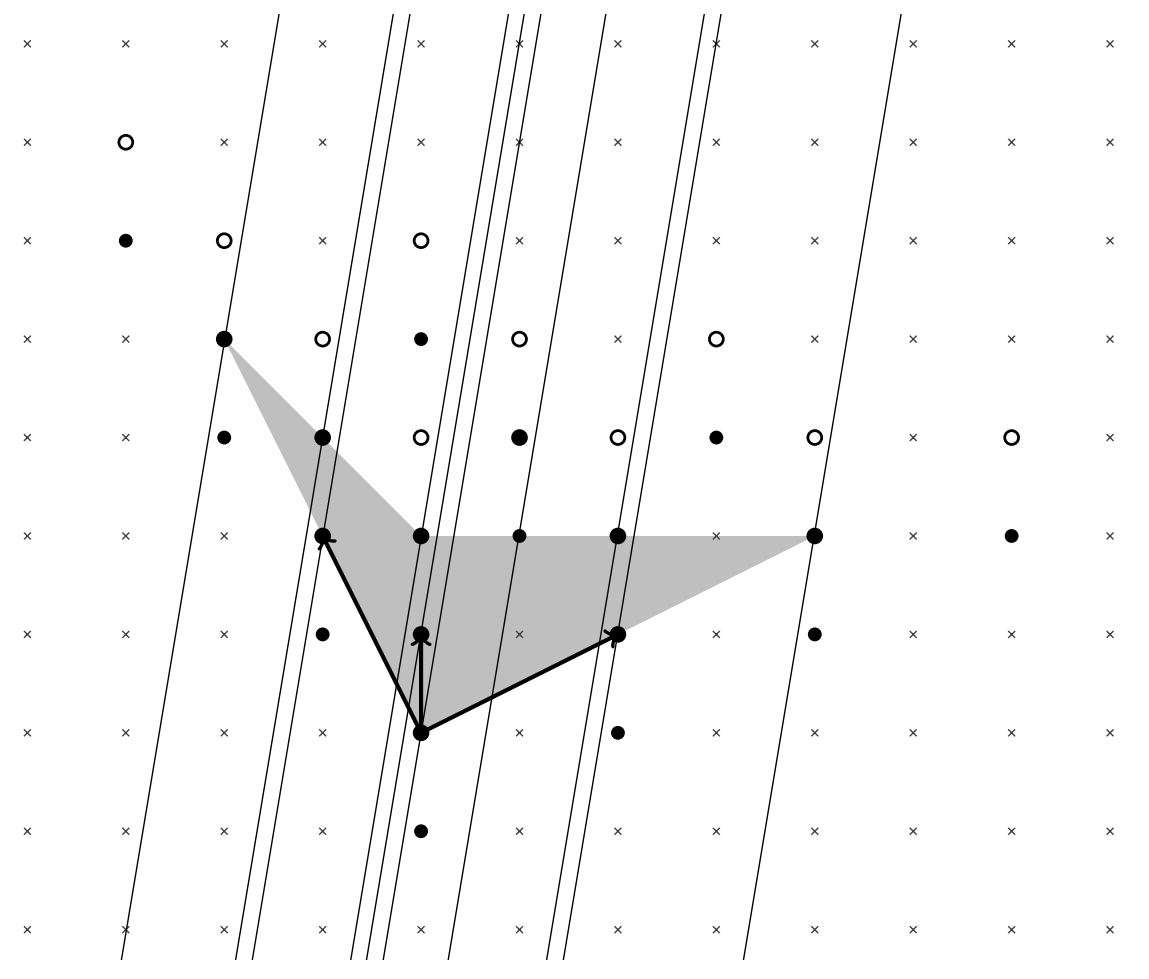

Figure 2: The case $\gamma=1$ of lemma 4.2 for a cone generated by three vectors, $a_{0}^{\mathrm{T}}=(0,1), a_{1}^{\mathrm{T}}=(-1,2), a_{2}^{\mathrm{T}}=(2,1)$. The region $Q$ is shaded in gray, the points of $V^{*}$ are represented as filled circles, the points of $V \backslash V^{*}$ are drawn as empty circles, and hyperplanes $H_{b}$ for each $b \in V^{*} \cap Q$ are indicated. The integer point $(1,1)$ lies in $Q \backslash V^{*}$, so the generating vectors do not form a 1-relaxed Hilbert basis for this cone.

points. SIAM Review, 10(3):371-372, 1968.

[21] G. Zambelli. Colorings of $k$-balanced matrices and integer decomposition property of related polyhedra. Operations Research Letters, 35(3):353-356, 2007. 